\title{
Prevalência de enteroparasitose em comunidade ribeirinha do estado do Pará, Brasil
}

\author{
Prevalence of enteroparasitosis in a riverside community in the state of Pará, Brazil
}

Prevalencia de enteroparasitosis en una comunidad ribereña en el estado de Pará, Brasil

Luann Wendel Pereira de Sena ${ }^{1,2 *}$, Cintia de Sousa Costa Pantoja ${ }^{1}$, Denise Anne Silva de Souza ${ }^{1}$, Suellen Silva Reis Palheta ${ }^{1}$, Amanda Gabryelle Nunes Cardoso Mello², José Luiz Fernandes Vieira², José Eduardo Gomes Arruda², Maria Pantoja Moreira ${ }^{3}$.

\begin{abstract}
RESUMO
Objetivo: Este trabalho tem como objetivo, investigar a prevalência de enteroparasitoses em populações ribeirinhas do estado do Pará, Brasil. Métodos: No período de julho a outubro de 2018, foram coletadas 104 amostras fecais de pessoas de ambos os sexos com idade de 2 a 75 anos e conduzidas ao Laboratório de Análises Clínicas da Escola Superior da Amazônia para análise parasitológica seguindo a metodologia de Hoffman, Pons e Janer. A positividade das amostras foi confirmada pela visualização de formas evolutivas de enteroparasitas. Resultados: Os resultados revelaram positividade em $95,1 \%$ dos casos, ressaltando que em $23 \%$ eram de poliparasitados. Os helmintos foram os mais frequentes, destacando-se Ascaris lumbricoides (44,23\%) e Enterobius vermiculares (13,46\%). Entre os protozoários, os mais frequentes foram Entamoeba histolytica/díspar (24,05\%), Entamoeba coli (7,69\%). Conclusão: Foi encontrado elevado número de casos de parasitismo intestinal na área de estudo, o que recomenda de políticas públicas voltadas à melhoria das condições de saúde das comunidades ribeirinhas.
\end{abstract}

Palavras-chave: Doenças parasitárias, Helmintos, Infecções por protozoários.

\begin{abstract}
Objective: The present study aimed to verify the prevalence of enteroparasites in riverside populations in the State of Pará, Brazil. Methods: This is a prospective study of cases of individuals suspected of having enteroparasites. A total of 104 stool samples were analyzed using the Lutz and Hoffman method to search for parasitic agents. Results: The results revealed positivity in $95.1 \%$ of the cases, emphasizing that in $23 \%$ they were multiparasitic. Helminths were the most frequent, with Ascaris lumbricoides (44.23\%) and Enterobius vermicular (13.46\%) standing out. Among the protozoa, the most frequent were Entamoeba histolytica / disparate (24.05\%), Entamoeba coli (7.69\%). Conclusion: A high number of intestinal parasitism cases was found in the study area, which recommends public policies aimed at improving the health conditions of riverside communities.
\end{abstract}

Keywords: Parasitic diseases, Helminths, Protozoan infections.

\section{RESUMEN}

Objetivo: El presente estudio tuvo como objetivo verificar la prevalencia de enteroparásitos en las poblaciones ribereñas en el estado de Pará, Brasil. Métodos: Este es un estudio prospectivo de casos de individuos sospechosos de tener enteroparásitos. Se analizaron un total de 104 muestras de heces utilizando el método de Lutz y Hoffman para buscar agentes parásitos. Resultados: Los resultados revelaron positividad en el

${ }^{1}$ Escola Superior da Amazônia (ESAMAZ), Belém - PA. *E-mail: luannsena@gmail.com

¿Universidade Federal do Pará (UFPA), Belém - PA.

${ }^{3}$ Secretária Municipal de Saúde de Belém (SESMA), Belém - PA.

SUBMETIDO EM: 7/2020

ACEITO EM: 8/2020

PUBLICADO EM: 11/2020 
$95.1 \%$ de los casos, enfatizando que en el $23 \%$ fueron multiparasitarios. Los helmintos fueron los más frecuentes, destacando Ascaris lumbricoides (44.23\%) y Enterobius vermobular (13.46\%). Entre los protozoos, los más frecuentes fueron Entamoeba histolytica / disparate (24.05\%), Entamoeba coli (7.69\%). Conclusión: Se encontró una gran cantidad de casos de parasitismo intestinal en el área de estudio, que recomienda políticas públicas destinadas a mejorar las condiciones de salud de las comunidades ribereñas.

Palabras clave: Enfermedad parasitaria, Helmintos, Infeciones por protozoos.

\section{INTRODUÇÃO}

As parasitoses intestinais, causadas por protozoários e helmintos, afetam grande parte da população mundial (WHO, 2020). Segundo uma estimativa da Organização Mundial de Saúde (OMS), em 2010, aproximadamente um terço da população vivia em condições ambientais que facilitavam a disseminação das infecções, afetando 3,5 bilhões de pessoas no mundo, com 450 milhões de doentes e resultando em 65 mil óbitos por ano (SARMENTO RR, 2014).

As condições socioeconômicas, de moradia e saneamento básico são determinantes na transmissão e propagação desses parasitos, os quais são transmitidos através da ingestão de água e alimentos contaminados, através do contato de fezes de humanos e animais infectados por esses microrganismos, e/ou pela penetração de larvas na pele, presentes no solo contaminado (BRAGAGNOLLO GR, et al., 2018).

As condições insatisfatórias, ou a inexistência de saneamento básico, aliados às precárias condições de vida das populações mais pobres, são responsáveis direto pela elevada prevalência das parasitoses intestinais nestas populações (SOARES JS, et al., 2013). Além disso, entende-se que o desconhecimento dos princípios básicos de higiene pessoal e de cuidados na manipulação durante o preparo dos alimentos, facilitam a proliferação de infecções parasitárias e predispõe os sujeitos a reinfecções nas áreas endêmicas (ANDRADE EC, et al., 2010).

Do ponto de vista da saúde pública, as helmintoses e protozooses intestinais representam grave problema, que leva ao prejuízo da função de alguns órgãos vitais, contribuindo para o aumento da desnutrição. Já com relação ao social, essas afecções possuem implicação direta na diminuição da qualidade de vida da população e causam consideráveis perdas econômicas, em função da redução da produtividade (WIEBBELLING AM, et al., 2015).

A interação entre o agente, o meio ambiente e o hospedeiro humano suscetível são responsáveis pela transmissão e manutenção da doença parasitária na população humana. O meio ambiente tem grande contribuição na interação parasita-hospedeiro e em todo os tipos de ambientes podem-se encontrar os prérequisitos para transmissão da infeção parasitária (ANTUNES AS e LIBARDONI KS, 2017). Além disso, a prevalência das parasitoses intestinais depende essencialmente do grau de exposição do indivíduo às formas infectantes dos parasitas (cistos, ovos ou larvas) (DE CARLI GA., 2006).

Ressalta-se ainda, que o ambiente inclui os fatores sociais, políticos e econômicos e, que a estrutura política e a situação econômica determinam, parcialmente, o estado nutricional dos membros de uma sociedade. Assim, essas parasitoses apresentam variações inter e intrarregionais relacionadas com o índice de aglomeração da população, com as condições de uso e contaminação do solo, da água e dos alimentos, e com a capacidade de evolução das larvas e ovos de helmintos e cistos de protozoários em cada um desses ambientes (ANDRADE EC, et al., 2010).

As parasitoses são endêmicas em populações de baixa renda, sendo considerada negligenciada, por apresentarem investimentos reduzidos no controle, nas pesquisas e na produção de medicamentos. Desse modo, apresenta elevada prevalência em países subdesenvolvidos e em desenvolvimento, tendo como consequência altos índices de morbidade na população infantil dado os efeitos debilitantes que podem causar alterações sobre o estado nutricional e no desenvolvimento físico e cognitivo (MACCHIONI F, et al., 2015).

Fatores clássicos, como a ausência de saneamento básico, favorece a transmissão e o consequente aumento significativo da frequência dessas doenças. Assim, em países subdesenvolvidos, uma grande parcela da população vive em precárias condições de saneamento, o que está relacionado ao meio ambiente, 
a qualidade precária de habitação, ao tipo de solo e variações climáticas, proporcionando uma maior prevalência destas em comunidades ribeirinhas que habitam regiões da floresta amazônica (MONTEIRO AC, et al., 2017).

Sabe-se que somente cerca de $60 \%$ da população brasileira detêm serviços de água encanada e $45 \%$ possuem saneamento básico necessário (MONTEIRO AC, et al., 2017). A água pode funcionar como importante veiculador de ovos de helmintos e cistos de protozoários, considerando a indisponibilidade de água potável para toda a população, é comum a utilização de formas alternativas de abastecimento doméstico, como por exemplo a utilização de água proveniente de cisternas, minas, lagos e rios (SILVA AM, et al., 2014).

O consumo de água de poços e de rios são comuns entre as comunidades ribeirinhas da Amazônia, onde muitas vezes os poços são escavados sem proteção em suas paredes laterais, tonando-se suscetível à contaminação, aumentando consideravelmente o risco de doenças parasitárias, entre outras de veiculação hídrica, nestas localidades (BAPTISTA AB, et al., 2014).

Embora, as comunidades ribeirinhas façam parte da realidade regional amazônica, elas encontram-se muito longe da realidade cotidiana da academia. Essas comunidades são desprovidas de recursos básicos. A precariedade de desenvolvimento está relacionada ao difícil acesso, que culmina no isolamento dessas populações. Por conta dessas dificuldades, o número de profissionais dispostos a trabalhar nessas áreas é escasso, o que torna insuficiente as referências sobre o tema.

Agravando o problema, os projetos governamentais efetivos para o desenvolvimento dessas comunidades são limitados, e muitas vezes não passam de iniciativas que contemplam questões imediatas e a obtenção de recursos à curto prazo (GONÇALVES RM e DOMINGOS IM, 2019).

Apesar de, as adversidades de acesso aos serviços básicos de saúde, os ribeirinhos consideram que a saúde local é "satisfatória", pois detêm a proximidade da natureza, ar puro, pouco barulho e bem-estar individual e social (GONÇALVES RM e DOMINGOS IM, 2019). Com base nestas informações, este estudo teve como objetivo analisar a prevalência de enteroparasitoses em uma comunidade ribeirinha do Estado do Pará, Brasil, visando obter-se melhor conhecimento dessas afecções nesta população.

\section{MÉTODOS}

Trata-se de um estudo prospectivo, quantitativo e transversal de casos destinado a determinar a prevalência de enteroparasitoses intestinais durante o período de julho de 2018 a outubro de 2018 em uma comunidade ribeirinha do Estado do Pará, Brasil.

Os pacientes da comunidade ribeirinha residiam em casas de madeira instaladas à margem do rio. Os critérios de inclusão no estudo foram: pacientes de ambos os sexos com idades de 2 anos a 75 anos, que não fizeram o uso de antiparasitários nos últimos 30 dias e que concordaram espontaneamente em participar do estudo e as crianças que foram autorizadas por seus país e/ou responsáveis, após a leitura e assinatura do Termo de Consentimento Livre e Esclarecido (TCLE). Foram excluídos deste estudo crianças que não foram autorizadas por seus país e/ou responsáveis a participarem e pacientes que não estejam na faixa etária de 2 anos a 75 anos e que não concordaram em assinar o TCLE.

As coletas das amostras de fezes foram realizadas em três dias alternados para cada paciente. As fezes foram coletadas em frascos coletores apropriados contendo formol a 10\% e acondicionadas em recipientes refrigerados para o transporte até o laboratório de análises clínicas de uma escola do ensino superior do estado do Pará, onde foram realizadas as análises laboratoriais.

As análises laboratoriais foram realizadas pelo método de Lutz (1919) ou de Hoffman (1934). O procedimento equivaleu basicamente na mistura do material fecal com água, filtração por gaze cirúrgica, repouso, até a formação da sedimentação. A sedimentação ocorreu pela inserção em três lâminas para cada amostra fecal e os resultados negativos foram refeitos. Após a obtenção dos resultados positivos, os pacientes foram direcionados a Unidade Básica de Saúde da comunidade ribeirinha de São Domingos do Baixo Acará, para serem submetidos ao tratamento médico e terapêutico específico. 
Após as análises parasitológicas, os dados foram armazenamento em planilhas do Microsoft Excel, organizado em categorias, respostas e resultados do exame, para serem posteriormente avaliados estatisticamente pelo Software BioEstat 5.0, estabelecendo o nível de significância de $95 \%$ ( $p>0.05$ ).

O estudo foi autorizado pelo Comitê de Ética em Pesquisa da Faculdade Integrada Brasil Amazônia (FIBRA), (CAAE): 95608418.0000.8187, tomando como base a Resolução 466/12 do Conselho Nacional de Saúde (CNS), que fornece os subsídios necessários para o desenvolvimento de pesquisas envolvendo seres humanos.

Inicialmente todos os sujeitos foram orientados a respeito da realização da pesquisa, e aqueles que aceitaram participar do presente estudo foram convidados a assinarem o TCLE. Ainda, toda a população de estudo foi informada acerca do objetivo da pesquisa e dos procedimentos necessários para obtenção das amostras e análise dos resultados, em que ficariam com os resultados e seriam orientados, caso positivo, a procurarem atendimento especializado para ter o tratamento adequado e estabelecerem uma educação em saúde sobre a diversas formas de contaminação por enteroparasitas.

\section{RESULTADOS}

Um total de 104 pacientes foram recrutados para o estudo e 312 amostras de fezes foram coletadas e analisadas. Também foi observado o perfil socioeconômico dos sujeitos que participaram do estudo (Tabela 1).

Tabela 1 - Perfil socioeconômico dos sujeitos que participaram do estudo.

\begin{tabular}{|c|c|}
\hline Características & Pacientes $(n=104)$ \\
\hline Sexo masculino $(\%, n)$ & $64,42(67)$ \\
\hline Idade (anos) & $27(2-75)$ \\
\hline Peso (Kg) & $57,6(7-72)$ \\
\hline \multicolumn{2}{|l|}{ Cor da Pele } \\
\hline Branca $(\%, n)$ & $7,69(8)$ \\
\hline Negro $(\%, n)$ & $21,15(22)$ \\
\hline Pardo $(\%, n)$ & $71,15(74)$ \\
\hline \multicolumn{2}{|l|}{ Escolaridade } \\
\hline Alfabetizadas $(\%, n)$ & $75,96(79)$ \\
\hline Não alfabetizadas (\%, n) & $9,61(10)$ \\
\hline Ensino Médio/Superior (\%,n) & $14,42(15)$ \\
\hline \multicolumn{2}{|l|}{ Renda Familiar } \\
\hline 1 Salário mínimo $(\%, n)$ & $100(104)$ \\
\hline Instalações sanitárias fora do domicílio (\%, n) & $100(104)$ \\
\hline Infecção por mais de 1 parasita $(\%, \mathrm{n})$ & $23,07(24)$ \\
\hline
\end{tabular}

Legenda: *resultados são expressos como média (desvio padrão) e percentagem.

Fonte: Sena, et al., 2018.

$\mathrm{Na}$ análise deste perfil, do total de entrevistados, $64,4 \%$ eram do sexo masculino, de cor parda e 75,9\% são alfabetizados. Quanto à renda mensal familiar, todos afirmaram ganhar um salário mínimo. Ao avaliar as condições de moradia e saneamento desta população, foi possível observar que todas as instalações sanitárias estavam situadas fora das moradias, sendo que o maior percentual (39\%) foi referente aos domicílios que possuía fossas a céu aberto, seguido de fossa escada $(31,7 \%)$ e de madeira $(28,5 \%)$.

Com relação aos resultados dos exames coproparasitológico, dos 104 pacientes que realizaram, 95,1\% foram positivos, com $23 \%$ poliparasitados, em que a espécie Ascaris lumbricoides $(44,2 \%)$ foi a mais prevalente, seguida de Enterobius vermiculares (13,4\%). Entre os protozoários prevaleceram Entaboeba histolytica/Entamoeba díspar (24\%) e Entamoeba coli (7,6\%) (Tabela 2). 
Tabela 2 - Distribuição das espécies enteroparasitárias obtidas na análise coproparasitológica da população estudada em uma comunidade ribeirinha do Estado do Pará, Brasil.

\begin{tabular}{ccc}
\hline Espécies Parasitárias & $\mathbf{n}$ & $\%$ \\
\hline Helmintos* & & \\
\hline Ascaris lumbricoides & 46 & 44,23 \\
Enterobius vermiculares & 14 & 13,46 \\
Ancilostomideos & 6 & 5,76 \\
Trichuris trichiura & 5 & 4,81 \\
\hline Protozoários & \\
\hline Entamoeba histolytica/Entamoeba díspar & 32 & 24,05 \\
Entamoeba coli & 9 & 7,69 \\
\hline
\end{tabular}

Legenda: ${ }^{*} X^{2}=64.06 p<0.001 ;{ }^{* *} X^{2}=11.8 p=0.0006$.

Fonte: Sena, et al., 2018.

Ao correlacionar a prevalência das infecções com a faixa etária, observa-se que as crianças de 2 a 10 anos (40,7\%) são as que apresentaram mais enteroparasitas quando comparado com os adultos (Tabela 3). E a faixa que apresentou mais poliparasitos na análise coproparasitológica foi de 44 a 54 anos, totalizando $8 \%$ (Tabela 4).

Tabela 3 - Distribuição de enteroparasitos de acordo com a faixa etária em uma comunidade ribeirinha do Estado do Pará, Brasil.

\begin{tabular}{cc}
\hline Prevalência de enteroparasitos por Idade \\
\hline Idade (anos) & $\%$ \\
\hline 02 a 10 & 40,79 \\
11 a 21 & 17,10 \\
22 a 32 & 9,21 \\
33 a 43 & 7,90 \\
44 a 54 & 14,47 \\
55 a 65 & 1,32 \\
66 a 75 & 1,32 \\
\hline
\end{tabular}

Fonte: Sena, et al., 2018.

Tabela 4 - Prevalência por idade de poliparasitados na análise coproparasitológica da população estudada em uma comunidade do Estado do Pará, Brasil.

\begin{tabular}{cc}
\hline Prevalência de Poliparasitismo por Idade \\
\hline Idade (anos) & $\%$ \\
\hline 2 a 10 & 2,5 \\
11 a 21 & 4,57 \\
22 a 32 & 2 \\
33 a 43 & 3 \\
44 a 54 & 8 \\
55 a 65 & 3 \\
66 a 75 & 0 \\
\hline
\end{tabular}

Fonte: Sena, et al., 2018.

\section{DISCUSSÃO}

A elevada prevalência de enteroparasitoses na comunidade ribeirinha localizada no Estado do Pará, Brasil, retrata a precária realidade de saúde das populações ribeirinhas, que se prolonga com o tempo, sem que sejam observadas mudanças significativas no panorama das doenças parasitárias nessas comunidades, levando a diminuição de qualidade de vida, perdas econômicas e sociais e alguns óbitos. Coura JR et al., (1993a) relatou a prevalência de $81,9 \%$ de parasitas intestinais em ribeirinhos do médio Solimões. Outro estudo do mesmo autor estabeleceu na comunidade de Saubinha uma prevalência de $95,1 \%$, na comunidade de Campinas 87,5\%, em Laranjal 83,1\% e em Damião 81,3\% (COURA JR, et al., 1993b). 
Em estudos realizados no médio Rio Negro, a prevalência de enteroparasitoses foi de 75,5\% (COURA CF, et al., 1994). Esteves NM (2005), na comunidade ribeirinha de Jutuba, no município de Belém, detectou uma prevalência de $73,3 \%$. Prevalência de $81,2 \%$ de parasitose intestinal foi constatado nos estudos realizados com os ribeirinhos do rio Piramanha, na llha das Onças, em Barcarena-PA (LOPES FM, et al., 2005).

As elevadas taxas de prevalência intestinal em comunidades ribeirinhas informadas por estes estudos corroboram com os resultados obtidos neste estudo e denotam a precariedade das condições de saneamento básico às quais essas comunidades estão submetidas, que associada ao baixo poder econômico e baixa escolaridade, contribuem para o cenário de caos instalado na saúde e na dignidade dessas populações.

Assim, a prevalência de parasitismo na região amazônica e em outros estados extra-amazônicos demonstram similaridade de achados com este estudo, como foi observado por Teixeira LM, et al., (2019), os quais constataram a presença de $76,31 \%$ das amostras de pacientes do município de Cametá, interior do estado do Pará, com resultado positivo para helmintos e/ou protozoários, em que 40 estavam infectadas por apenas um enteroparasita e 18 por dois ou mais parasitas.

Com isso, esse estudo e outros (DE LIMA OLIVEIRA, et al., 2019; GAMA, et al., 2018), denotam a precariedade das condições de saneamento básico às quais essas comunidades estão submetidas, que associada ao baixo poder econômico e baixa escolaridade, contribuem para o cenário de caos instalado na saúde e na dignidade dessas populações.

Os exames coproparasitológicos evidenciaram maior positividade enteroparasitária no sexo masculino $(64,42 \%)$. Gama, et al. (2018) demonstram que os homens são mais acometidos por doenças crônicas e muitas vezes severas em comparação as mulheres, devido serem muito mais relapsos e despreocupados com sua higiene pessoal, por exemplo, e não praticarem hábitos essenciais, como lavar as mãos antes das refeições.

Conforme ficou demonstrado neste estudo, a faixa etária mais atingida por enteroparasitas foi a de 02 a 10 anos $(40,79 \%)$. Sabe-se que os parasitas intestinais afetam indivíduos de todas as faixas etárias, mas as crianças são, via de regra, as mais atingidas, especialmente no Brasil, em que o número de indivíduos com algum tipo de enteroparasitose está elevado na população pediátrica ( $0-5$ anos) e crianças em idade escolar, principalmente (LOPES FM, et al., 2005; SILVA VF., et al. 2006).

Alguns autores atribuem este fato à maior exposição infantil à contaminação, pois esta população fica um maior tempo em contato com o solo, onde desenvolvem suas atividades lúdicas, e ainda não incorporam os princípios básicos de higiene (MAN B e CIMERMAN S, 1999; NEVES DP, 2005).

De fato, um estudo feito em escolas distrito de Martinésia (Uberlândia, MG) mostra que, entre 103 crianças examinadas, o coeficiente geral de prevalência foi de $22,3 \%$, com índices de infecção mais elevados no grupo etário de 8 a 9 anos $(34,8 \%)$ e no sexo feminino (26,9\%). Helmintoses e protozooses apresentaram taxas de prevalência similares (10,7\% e 12,6\%, respectivamente) (FERREIRA CB e MARÇAL-JUNIOR O, 1997).

Segundo Neves DP (2005), as mudanças de hábito associada ao desenvolvimento e amadurecimento do sistema imune, levam a redução progressiva das taxas de incidência e prevalência de parasitoses intestinais em adultos. Neste estudo, tal fato ficou evidenciado, uma vez que as prevalências de enteroparasitoses observadas nas faixas etárias mais avançadas atingiram níveis de apenas 1,32\%.

As taxas significativas de poliparasitismo na comunidade ribeirinha de estudo coincidem com as observadas pelos autores acima citados. O estudo demonstrou uma maior frequência de poliparasitismo em pacientes com faixa etária de 44 a 54 anos (8\%), quando comparadas a idade infantil (02 a 10 anos). Isso se justifica pelo fato de os adultos estarem mais expostos devido suas atividades laborais, como a pesca, característico da população da comunidade ribeirinha (SAMPIETRO V, et al., 2013).

Além disso, as taxas significativas de prevalência de poliparasitismo observadas, refletem o grau de exposição dessa comunidade ao contato com os parasitos e, portanto, o maior risco de desenvolver quadros graves, processos obstrutivos e formas invasivas de doenças parasitárias. 
No Brasil, os parasitas Entamoeba coli, Trichuris trichiura, Ascaris lumbricoides, Ancilostoma sp, Endolimax nana e Entamoeba hystolitica são tidos como os mais prevalentes, comprovado no estudo de Baptista, et al., (2006), que observou a incidência de parasitoses intestinais na Paraíba no período entre 1999 e 2004, detectando estes parasitas em $81,2 \%$ das amostras positivas.

De fato, os helmintos foram mais prevalentes em relação aos protozoários, com maior prevalência para o Ascaris lumbricoides, seguido de Enterobius vermiculares, o que pode estar correlacionado com o perfil epidemiológico da comunidade e a presença características ambientais sanitárias marcantes, como condições precárias de saneamento básico, presença de clima quente e úmido e solo arenoso, os quais contribuem ao desenvolvimento do ciclo biológico dos parasitas (VIEIRA EK, 2017; RODRIGUES JMN, et al., 2019; NUNES MO e ROCHA TJM, 2019).

Segundo Sampietro V, et al (2013), as parasitoses intestinais transmitidas por água e/ou alimentos contaminados, como A. lumbricoides, E. histolytica/díspar e G. lamblia, são mais prevalentes do que as transmitidas por penetração ativa de larvas na pele, como os Ancilostomídeos sp. e os S. stercoralis.

As doenças parasitárias intestinais constituem um indicativo sobre a situação de saúde das populações. A Amazônia legal abriga o maior contingente populacional rural do país, porém muito pouco se conhece da situação de saúde desta região, e isto se deve ao fato das principais pesquisas sobre o assunto estarem concentradas em dois grandes segmentos: as capitais (Belém, Santarém, Manaus, Boa Vista, Rondônia e Rio Branco) e as populações indígenas (PEREIRA G, et al., 2018; SOUZA FR, et al., 2018). Soma-se a isso, os desafios de equilibrar desenvolvimento econômico na região com a conservação da natureza, inclusive as dificuldades de se assegurar a sustentabilidade por meio da integração do mercado.

Outro fato é que pouco se conhece sobre outro segmento populacional da Amazônia: o campesinato (populações rurais não indígenas, caboclos e ribeirinhos), que constituem um percentual expressivo da população da região, mas que figuram como "população invisível" como muito bem definiu o antropólogo britânico Stephen Nugent S (1993). Assim, o saber tradicional e o saber científico devem ser aliados no combate à proliferação de doenças parasitárias, as quais estão correlacionadas com as condições socioeconômicas e sanitárias da região, cultivando, a cada vez mais a educação em saúde nas comunidades ribeirinhas.

Além disso, é necessário também o comprometimento comunitário para implantação, desenvolvimento e sucesso dos programas de controle das parasitoses, somado ao monitoramento médico dos indivíduos parasitados e que estão em risco de contaminação e acompanhamento mais próximo da introdução de terapia medicamentosa aos pacientes confirmados.

Também requer um incentivo constante na aplicação da educação em saúde pública e manipulação adequada de alimentos, como boa assepsia das mãos, quando houver contato com o solo ou ao usar o banheiro, e a lavagem adequada das frutas e verduras antes de consumi-las, visto que são medidas simples que previnem o aparecimento das parasitoses intestinais.

Além disso, a utilização de agentes sanitizantes eficazes (hipoclorito de sódio) na superfície de em frutas e verduras, capazes de eliminar estruturas parasitárias. Essas recomendações demonstram que apenas o diagnóstico clínico e laboratorial não é suficiente para controle e diminuição das parasitoses intestinais nesta região.

\section{CONCLUSÃO}

A prevalência de enteroparasitoeses nos indivíduos foi de 95,1\%, havendo predomínio dos parasitos Ascaris lumbricoides (44,2\%), Entamoeba histolytica/díspar (24,5\%) e Enterobius vermiculares (13,4\%). Com relação a faixa etária, a mais atingida por enteroparasitas foi a de 02 a 10 anos (40,79\%), em que estão mais expostos à contaminação pelo maior contato com ambientes infectados por larvas e/ou cistos de parasitas intestinais. Entretanto, a que apresentou uma maior frequência de poliparasitismo foi a faixa etária de 44 a 54 anos (8\%), os quais podem ter uma elevada taxa de infecção correlacionada as atividades laborais que desempenham nas comunidades ribeirinhas, como o extrativismo. Mesmo com esforços da saúde pública em 
combater enteroparasitos, esta doença ainda se apresenta como um grave problema de saúde pública nas populações ribeirinhas da Amazônia, uma vez que pessoas contaminadas são potentes vias de disseminação. Contudo, recomenda-se a inclusão de políticas públicas voltadas à melhoria das condições de saúde para estas comunidades, onde são muitas vezes esquecidas pelo poder público.

\section{AGRADECIMENTOS}

Agradecimento ao Laboratório de Análises Clínicas da Escola Superior da Amazônia (ESAMAZ) por colaborar na realização dos testes parasitológicos e à Comunidade Ribeirinha do Estado do Pará.

\section{REFERÊNCIAS}

1. ANDRADE EC, et al. Parasitoses intestinais: uma revisão sobre seus aspectos sociais, epidemiológicos, clínicos e terapêuticos. Rev APS. 2010;13(2): 231-240.

2. ANTUNES AS, LIBARDONI KSB. Prevalência de enteroparasitoses em crianças de creches do município de Santo Ângelo, RS. Revista Contexto \& Saúde. 2017; 17 (32): 144-156.

3. ARAUJO MM, et al. Prevalência de enteroparasitoses na região Norte de Mato Grosso. Nativa-Revista de Ciências Sociais do Norte de Mato Grosso. 2019; 8(2).

4. BAPTISTA AB, et al. Prevalência de enteroparasitos e aspectos epidemiológicos de crianças e jovens do município de altamira-pa/prevalence of intestinal parasites and epidemiological aspects of children and youth in the municipality of altamira, Pará, Brazil. Rev Pesq Saúde. 2013; 14(2): 77-80.

5. BAPTISTA SC, et al. Análise da incidência de parasitoses intestinais no município de Paraíba do Sul, RJ. Rev. Bras. Anal. Clin. 2006;38(4):271-273.

6. BRAGAGNOLLO GR, et al. Intervenção educacional sobre enteroparasitoses: um estudo quase experimental. Revista Cuidarte. 2018; 9(1): 2030-2044.

7. CAVALCANTE UMB, et al. Avaliação da Qualidade de Vida de pacientes com enteroparasitoses atendidos num Hospital Escola de João Pessoa-PB utilizando um Modelo de Regressão Logística. Tempus Actas de Saúde Coletiva. 2016; 10(2): 275-288.

8. CIMERMAN B, CIMERMAN S. Parasitologia humana e seus fundamentos gerais. São Paulo: Atheneu, 1999. Cap. 5, p. $10-21$

9. COURA JR, et al. Aspectos epidemiológicos, sociais e sanitários em áreas do Médio Solimões. I. Estudo nas localidades de São Francisco do Laranjal, Aranaí e São Lázaro do Surubim, Município de Coari, Amazonas. Anais da Academia Nacional de Medicina, 1993a; 153: 122-126.

10. COURA JR, et al. Aspectos epidemiológicos, sociais e sanitários em áreas do médio solimões. II. Estudo de dois bairros periféricos da cidade de Coari e quatro localidades do lago do Mamiá, Estado do Amazonas. Anais da academia nacional de medicina. 1993b; 153: 183-186.

11. COURA CF, et al. Aspectos epidemiológicos, sociais e sanitários de uma área no rio Negro, Estado do Amazonas, com especial referência as parasitoses intestinais e a infecção chagásica. Caderno de Saúde Pública. 1994; 10:331334.

12. DE CARLI GA. Parasitologia Clínica: Seleção de Métodos e Técnicas de Laboratório para o Diagnóstico das Parasitoses Humanas. São Paulo: Atheneu, 2006.

13. DE LIMA OLIVEIRA A, DE ARRUDA BARBOSA VS. Prevalência de Enteroparasitoses em usuários do laboratório de Análises Clínicas de Araruna-PB. Revista Saúde \& Ciência Online. 2019; 7(3): 5-22.

14. DE OLIVEIRA RODRIGUES PC, et al. Características socioeconômicas, demográficas e de saúde de escolares residentes em duas comunidades da Amazônia Meridional brasileira. Revista Ciência e Estudos Acadêmicos de Medicina, 2017; 1(06).

15. DOS SANTOS LIMA TM, et al. Estado da Arte sobre enteroparasitos em comunidades indígenas do Brasil. Espaço Ameríndio. 2017; 11(2): 274.

16. ESTEVES NM. Diagnóstico coproparasitológico e avaliação dos fatores de risco para infecção parasitária intestinal em uma comunidade ribeirinha do município de Belém - Pará - Brasil - Instituto de Ciências da Saúde. Universidade Federal do Pará, Belém, 2005; $100 \mathrm{p}$.

17. FERREIRA CB, MARÇAL JUNIOR O. Enteroparasitoses em escolares do Distrito de Martinésia, Uberlândia, MG: um estudo piloto. Rev. Soc. Bras. Med. Trop. 1997; 30:373-7.

18. GAMA ASM et al. Inquérito de saúde em comunidades ribeirinhas do Amazonas, Brasil. Cadernos de Saúde Pública. 2018; 34: e00002817.

19. GONÇALVES RM, DOMINGOS IM. População ribeirinha do Amazonas e a desigualdade no acesso à saúde. Revista de Estudos Constitucionais, Hermen6eutica e Teoria do Direito (RECHTD). 2019; 11(1):99-108

20. GOMES TM, et al. Pesquisa de enteroparasitas em meios de transporte público urbano da cidade de Patos de Minas, MG-Brasil. Psicologia e Saúde em debate. 2016; 2(1): 74-99.

21. HOFMANN WA, et al. The sedimentation concentration method in Schistosomiasis mansoni. PR J Public Health Trop Med. 1934; 9:283-91

22. LOPES FMR, et al. Aspectos coproparasitológicos em escolares do município de Jataizinho, Paraná. In: XIX Congresso Brasileiro de parasitologia, 19., 2005, Porto Alegre. Anais. CD ROM. 
23. MACCHIONI F, et al. Dramatic Decrease in Prevalence of Soil-Transmitted Helminths and New Insights into Intestinal Protozoa in Children Living in the Chaco Region, Bolivia. Revisit the American Journal of Tropical Medicine and Hygiene. 2015; 92(4): 794-796.

24. MONTEIRO ACS, et al. Prevalência e fatores associados à enteroparasitoses em escolares. 2017.

25. NEVES DP. Parasitologia Humana. 11 ed. São Paulo: Atheneu, 2005. 494 p.

26. NUGENT S. Amazonian Caboclo Society: na essay on invisibility and peasant economy. Oxford:Berg, 1993.

27. NUNES MO, ROCHA TJM. Fatores condicionantes para a ocorrência de parasitoses entéricas de adolescentes. Journal of Health \& Biological Sciences. 2019; 7(3): 265-270.

28. PEREIRA G, et al. Prevalência de infecções parasitárias intestinais oriundas de crianças residentes em áreas periféricas, município de Juazeiro do Norte-Ceará. Revista Interfaces: Saúde, Humanas e Tecnologia. 2018; 5(14): 21-27.

29. RODRIGUES JMN, et al. Prevalência de Enteroparasitoses Humanas no Município de Itacaré Estado da Bahia, no ano de 2018 e Fatores Agravantes/Prevalence of Human Enteroparasitosis in the City of Itacaré State of Bahia, year 2018 and Advancing Factors. Revista de Psicologia. 2019; 13 (48): 168-174.

30. SAMPIETRO V, et al. Enteroparasitoses e aspectos epidemiológicos na população geronte de uma unidade básica de saúde de Cascavel, Paraná. Rev Thêma Scientia. 2013;3(1):130-138.

31. SARMENTO RR. Comparação da prevalência de exames coprológicos, testes sorológicos e exames hematológicos e a associação entre a resposta imunológica por Th1, Th2 e Treg em idosos na região metropolitana de João Pessoa Paraíba. [Tese] Porto Alegre: Gerontologia Biomédica. Pontifícia Universidade Católica do Rio Grande do Sul. 2014.

32. SILVA AMB, et al. Ocorrência de enteroparasitoses em comunidades ribeirinhas do Município de Igarapé Miri, Estado do Pará, Brasil. Revista Pan-Amazônica de Saúde, 2014; 5(4): 45-51.

33. SILVA VF, et al. Epidemiologia das enteroparasitoses em crianças de idade pré-escolar no município de Guaraci, Paraná. In: XIV Congresso Brasileiro de Parasitologia Veterinária e II Simpósio Latino Americano de Riquetsioses. Anais. Ribeirão Preto, 2006. p. 394.

34. SOARES JS, et al. Educação e promoção em saúde para ex-moradores de rua. Em Extensão. 2013; $12(1)$ : $159-167$.

35. SOUZA FR, et al. Diagnóstico epidemiológico de parasitos intestinais em uma comunidade rural de Ipatinga-MG. Revista Uningá. 2018; 55(2): 200-213.

36. TEIXEIRA LMS, et al. Prevalência de enteroparasitose e sua associação com perfil hematológico e bioquímico em adultos em Cametá, Pará, Brasil. Infarma. 2019; 31(4): 293-304.

37. VASCONCELOS FG, et al. Percepção dos problemas ambientais em uma comunidade ribeirinha da região amazônica brasileira. Anais SNCMA. 2017; 8(1).

38. VIEIRA EK. Influência das estações seca e cheia na ocorrência das parasitoses intestinais no município de Tefé, Amazonas, Brasil. Trabalho de Conclusão de Curso (Licenciatura em Ciências Biológicas) - Universidade do Estado do Amazonas, Amazonas, 2017; 9p.

39. WIEBBELLING AMP, et al. Parasitoses intestinais em crianças de creches/escolas de Porto Alegre: prevalência e profilaxia. Raízes e Rumos. 2015;3(1):182-183.

40. WORLD HEALTH ORGANIZATION. Soil-transmitted helminth infections. 2020. 